Quim. Nova, Vol. 27, No. 1, 50-54, 2004

\title{
DESENVOLVIMENTO DE UM MICRO-AQUECEDOR PARA SISTEMAS DE ANÁLISE QUÍMICA EM FLUXO. DETERMINAÇÃO ESPECTROFOTOMÉTRICA DE MANGANÊS EM PLANTAS
}

\author{
Marisa Smiderle \\ Instituto de Química de São Carlos, Universidade de São Paulo, Av. Trabalhador São Carlense, 400, 13560-960 São Carlos - SP \\ Maria Cristina Tomazzini e Boaventura F. Reis* \\ Centro de Energia Nuclear na Agricultura, Universidade de São Paulo, Av. Centenário, 303, 13400-970 Piracicaba - SP
}

Recebido em 18/12/02; aceito em 15/5/03

\begin{abstract}
DEVELOPMENT OF A MICRO-HEATER FOR FLOW ANALYSIS SYSTEMS. SPECTROPHOTOMETRIC DETERMINATION OF MANGANESE IN PLANT DIGEST. In this work a micro-heater device to be used as an integral part of the flow analysis manifold is described. The usefulness of the device was demonstrated using it in the development of a multicommutated flow analysis procedure for the spectrophotometric determination of manganese in plant digest. The method was based on the manganese oxidation by periodate in phosphoric acid medium to form the permanganate anion. The reaction development is dependent on the temperature and it was observed that at $25^{\circ} \mathrm{C}$ a time interval of $c a .15$ min was necessary for the reaction to attain equilibrium. Setting the temperature to $70{ }^{\circ} \mathrm{C}$, this time interval could be decreased to $c a .30 \mathrm{~s}$. This condition was easily attained employing the proposed micro-heater device coupled to the manifold. The procedure was applied to manganese determination in soybean digests and results compared with those obtained by inductively coupled argon plasma optical emission spectrometry (ICP-OES). No significant difference at $90 \%$ confidence level was observed. A linear response for sample concentrations ranging from 5.0 to $30.00 \mathrm{mg} \mathrm{L}^{-1} \mathrm{Mn}^{2+}$; a relative standard deviation of $1.3 \%(\mathrm{n}=6)$ for a typical sample containing $6.3 \mathrm{mg} \mathrm{L}^{-1} \mathrm{Mn}^{2+}$; a sampling rate of 22 determinations per hour; a low reagent consumption, of $12.0 \mathrm{mg} \mathrm{NaIO}$ per determination; and a detection limit of $1.2 \mathrm{mg}$ $\mathrm{L}^{-1}$ were achieved.
\end{abstract}

Keywords: multicommutated flow system; micro-heater device; manganese determination.

\section{INTRODUÇÃO}

Em todos os procedimentos analíticos baseados no conceito de análise por injeção em fluxo (FIA) ${ }^{1}$, existe um compromisso entre alguns parâmetros do sistema e o desenvolvimento da reação. Quando a cinética da reação química envolvida é lenta, é necessário um longo tempo de residência a fim de se obter sensibilidade adequada. Por outro lado, um aumento no tempo de residência pode causar diluição da amostra, comprometendo o ganho de sinal. Visando minimizar este efeito, procedimentos analíticos empregando diferentes estratégias, como parada de fluxo ${ }^{2}$, retenção da zona da amos$\operatorname{tra}^{3}$ e fluxo monossegmentado por $\operatorname{ar}^{4}$, têm sido empregadas na busca de melhores condições de reação com o mínimo de dispersão.

No sentido de viabilizar o emprego de reações de cinética lenta, dispositivos de aquecimento também têm sido utilizados em sistemas FIA e, dentre esses, podemos citar o emprego de forno de microondas $^{5-7}$ e banho térmico usando água como fluido trocador de calor $^{8-10}$. Estes equipamentos, em geral, apresentam grandes dimensões, o que dificulta a montagem do módulo de análise.

Neste trabalho é proposto um dispositivo para aquecimento em sistemas de análise química em fluxo, o qual pode ser acoplado ao módulo de análise permitindo o controle da temperatura da bobina de reação em função da vazão do fluido transportador e da diferença de potencial aplicada, uma vez que o reator de aquecimento também é usado como bobina de reação. Para demonstrar a aplicabilidade do dispositivo desenvolveu-se um procedimento para determinação espectrofotométrica de manganês em digeridos de material vegetal, empregando o método baseado na oxidação de $\mathrm{Mn}^{2+}$ a $\mathrm{MnO}_{4}^{-}$pelo

*e-mail: reis@cena.usp.br periodato de sódio em meio de ácido fosfórico a quente ${ }^{11-12}$. O módulo de análise baseado no conceito de multicomutação $0^{13,14}$ permite controlar a inserção da amostra e do reagente e também a diferença de potencial aplicada ao aquecedor através da mesma interface de controle.

\section{PARTE EXPERIMENTAL}

\section{Equipamentos e acessórios}

Os equipamentos utilizados foram um espectrofotômetro Femto, modelo 435 equipado com cela de fluxo de volume interno de $180 \mu \mathrm{l}$ e passo ótico de $12 \mathrm{~mm}$; bomba peristáltica Ismatec, modelo IPC-8, equipada com tubos de bombeamento de "tygon"; microcomputador 486 equipado com uma interface eletrônica (PCL - 711S, Advantech Co.) para controlar o módulo de análise e efetuar a aquisição de dados através da porta de saída digital e da entrada analógica; fonte regulada de $12 \mathrm{~V}$ para acionamento das válvulas solenóides; relê de estado sólido (S505-0SJ610-000, Continental Industries, Inc.); um transformador de saídas de $12 \mathrm{~V}$ e corrente de 4 A; e um "dimmer" para ajuste de diferença de potencial.

O módulo de análise consiste de um conjunto de três válvulas solenóides de 3 vias (161T031, NResearch); um reator integrado ao sistema de aquecimento e linhas de fluxo de tubos de polietileno com diâmetro interno de $0,8 \mathrm{~mm}$.

\section{Soluções de reagentes e amostras}

Todas as soluções foram preparadas a partir de reagentes de grau analítico usando água destilada e purificada utilizando equipamento Milli-Q. 
Solução estoque de manganês $1000 \mathrm{mg} \mathrm{L}^{-1}$ foi preparada dissolvendo-se 0,7912 g de $\mathrm{MnO}_{2}$ em $20 \mathrm{~mL}$ de ácido clorídrico concentrado e completando-se o volume para $1000 \mathrm{~mL}$ com água.

Soluções padrão de manganês de 0,$0 ; 5,0 ; 10,0 ; 15,0 ; 20,0$ e $30 \mathrm{mg} \mathrm{L}^{-1} \mathrm{em}$ ácido perclórico $0,25 \mathrm{~mol} \mathrm{~L}^{-1}$ foram preparadas a partir da solução estoque de $\mathrm{Mn}^{2+} 1000 \mathrm{mg} \mathrm{L}^{-1}$.

Solução estoque de $\mathrm{KMnO}_{4}, 8,0 \mathrm{mmol} \mathrm{L}^{-1}$ foi preparada em $\mathrm{H}_{3} \mathrm{PO}_{4}$ $10 \%$ (v/v). Soluções de $\mathrm{NaIO}_{4}$ contendo 6,0; 8,0 e 10,0\% (m/v) foram preparadas em $\mathrm{H}_{3} \mathrm{PO}_{4} 0,5 \mathrm{~mol} \mathrm{~L}^{-1}$ contendo $\mathrm{KMnO}_{4} 0,36 \mathrm{mmol} \mathrm{L}$ 1

Amostras de soja (folhas e caule) foram mineralizadas empregando digestão nitro-perclórica, conforme descrito por Krug et al. ${ }^{15}$.

\section{Descrição do dispositivo de aquecimento}

O dispositivo de aquecimento, conforme mostra a Figura 1, é constituído por um fio de níquel-cromo de $0,5 \mathrm{~mm}$ de diâmetro interno, encapado com tubo de espaguete térmico usado para isolamento em fiação elétrica (tubo termocontráctil), o qual apresenta alta resistência ao ataque químico. O fio de níquel-cromo depois de encapado foi inserido em um tubo de "Teflon", fixado nas extremidades em dois blocos de acrílico. Uma diferença de potencial entre 5,0 e $10,0 \mathrm{~V}$ foi aplicada, dependendo do comprimento do fio. $\mathrm{O}$ aquecimento do fio de níquel-cromo causava o encolhimento do tubo de espaguete térmico, o que resultava em sua aderência ao fio. $\mathrm{O}$ diâmetro interno do tubo de "Teflon" utilizado era 1,6 mm e o diâmetro do fio de níquel-cromo com a capa protetora era 1,0 $\mathrm{mm}$. Assim, a solução era aquecida enquanto fluía através do espaço livre entre os dois tubos.

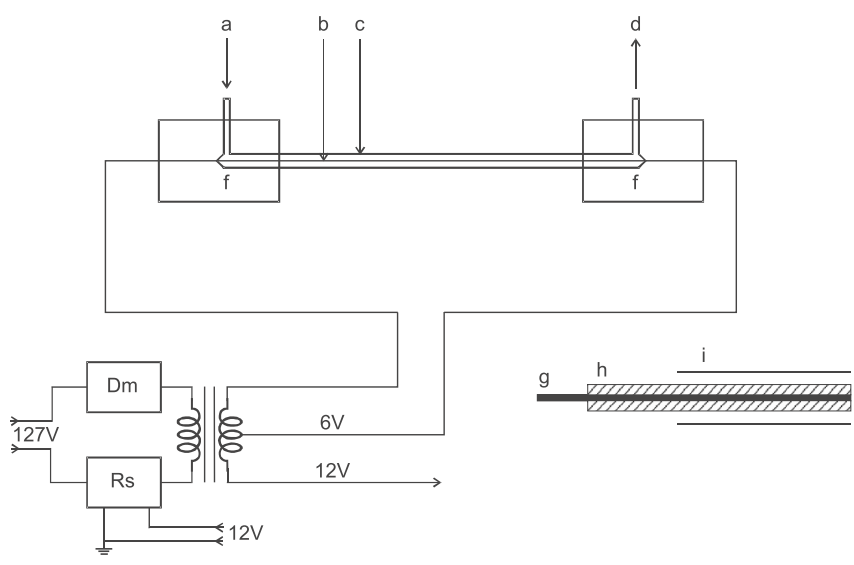

Figura 1. Desenho esquemático do aquecedor. a e d = entrada e saída de fluidos, respectivamente; $b=$ fio de níquel-cromo recoberto com capa quimicamente inerte; $c=$ tubo de Teflon, diâmetro interno $1,6 \mathrm{~mm} ; f=$ bloco de acrílico de $8 \times 6 \times 2 \mathrm{~cm} ; \mathrm{Dm}=$ dimmer; $R \mathrm{~s}=$ relê de estado sólido; $\mathrm{g}$, h e $i=$ vista em corte mostrando o fio de níquel-cromo, a capa protetora e o tubo de Teflon, respectivamente

O relê de estado sólido (Rs) é controlado pelo computador e pode ser programado para trabalhar no modo contínuo ou no modo intermitente. No primeiro modo, o mesmo é ligado no início do trabalho e desligado no encerramento. No modo intermitente, o relê é ligado durante um intervalo de tempo pré-determinado quando a zona da amostra estiver passando através do aquecedor.

Foram construídas 3 unidades do reator/aquecedor tendo comprimentos de 12,21 e $55 \mathrm{~cm}$, resistências de 0,$9 ; 1,7$; e $3,4 \Omega$ e volumes internos de 90, 200 e $460 \mu \mathrm{L}$, respectivamente. Visando verificar as respostas dos três reatores em função da diferença de potencial e da vazão do fluido, testes iniciais foram executados usando água como fluido. Para cada reator foram estabelecidas 3 diferenças de potenciais e a vazão de bombeamento foi variada de 0,9 a $8,2 \mathrm{~mL} \mathrm{~min}^{-1}$. Os experimentos foram efetuados em triplicata para verificar também a repetibilidade das medidas de temperatura. A determinação da temperatura foi feita utilizando um termômetro digital de um pHmetro sendo que a sonda de temperatura foi acoplada à saída do reator.

\section{Funcionamento do módulo de análise e estudo das variáveis do} sistema

O módulo de análise foi desenvolvido baseado no conceito de multicomutação, conforme mostra o diagrama de fluxo da Figura 2. Nessa configuração as válvulas estão desligadas, a solução transportadora (Ca) flui através do reator/aquecedor (B) em direção ao detector (Det) e as soluções da amostra e do reagente são desviadas para recuperação $(\operatorname{Re})$.
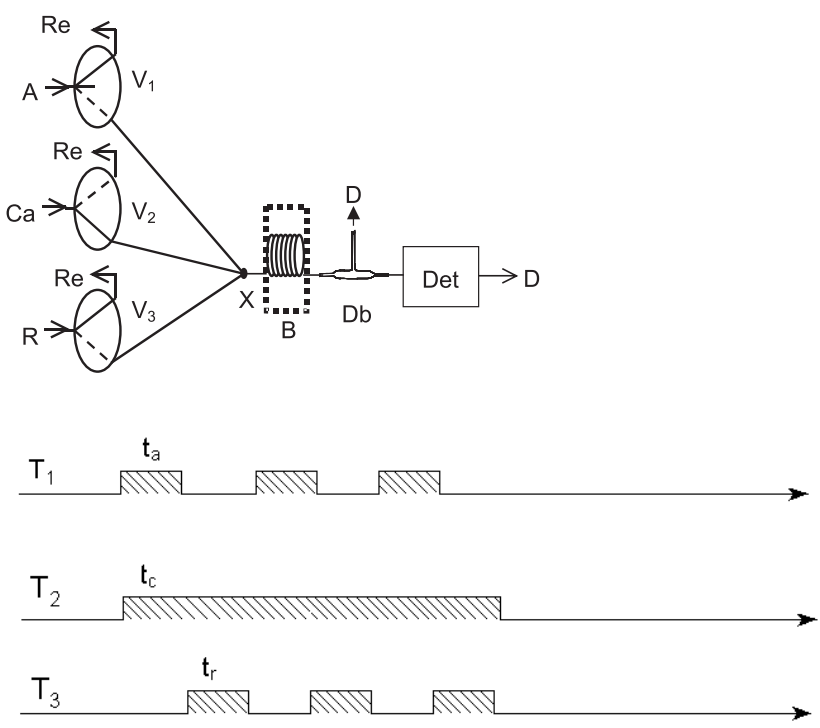

Figura 2. Diagrama de fluxo do módulo de análise. $V_{l}, V_{2}, V_{3}=$ válvulas solenóides de três vias; $B=$ micro-aquecedor $(460 \mu \mathrm{L} ; 3,4 \Omega)$; Det $=$ espectrofotômetro $(\lambda=548 \mathrm{~nm}) ; X=$ ponto de confluência; $A=$ amostra, vazão de 2,0 $\mathrm{mL} \mathrm{min}{ }^{-1}$; Ca = solução transportadora, vazão de 2,0 $\mathrm{mL} \mathrm{min}^{-1}$; $R=\mathrm{NaIO}_{4} 8,0 \%(\mathrm{~m} / \mathrm{v})$ contendo $\mathrm{MnO}_{4}^{-} 0,36 \mathrm{mmol} \mathrm{L}^{-1}$ em $\mathrm{H}_{3} \mathrm{PO}_{4} 0,5 \mathrm{~mol} \mathrm{~L}^{-1}$, vazão de 1,0 $\mathrm{mL} \mathrm{min}^{-1} ; R e=$ recuperação de soluções; $\mathrm{D} b=$ desborbulhador, vazão de 1,0 $\mathrm{mL} \mathrm{min} \min ^{-1}(100 \mu \mathrm{L}) ; D=$ descarte. $T_{1}, T_{2}$ e $T_{3}=$ diagrama de tempo de acionamento das válvulas solenóides $V_{1}, V_{2}, V_{3}$, respectivamente; $t_{a}=$ tempo de inserção da amostra; $t_{r}=$ tempo de inserção do reagente; $t_{c}=$ tempo de interrupção do bombeamento da solução transportadora. As linhas cheias nos símbolos das válvulas indicam a passagem das soluções quando a válvula está desligada e as linhas tracejadas, a passagem das soluções quando a válvula está ligada. As superfícies hachuradas no diagrama de tempos indicam que a respectiva válvula está acionada

Na etapa de amostragem, o microcomputador aciona as válvulas seqüencialmente seguindo a estratégia indicada no diagrama de tempo de acionamento das válvulas. Observa-se na Figura 2, que durante a etapa de amostragem a válvula $\mathrm{V}_{2}$ permanece constantemente ligada, enquanto as válvulas $\mathrm{V}_{1}$ e $\mathrm{V}_{3}$ são acionadas de forma alternada. Desta forma, alíquotas das soluções da amostra (A) e do reagente (R) são inseridas no reator/aquecedor. $\mathrm{O}$ volume de cada alíquota é dado pela equação $v_{\mathrm{a}}=\varphi_{\mathrm{a}} \mathrm{t}_{\mathrm{a}}$ e $\mathrm{v}_{\mathrm{r}}=\varphi_{\mathrm{r}} \mathrm{t}_{\mathrm{r}}$, onde $\varphi_{\mathrm{a}}$ e $\varphi_{\mathrm{r}}$ são as vazões da amostra e do reagente e $t_{\mathrm{a}}$ e $\mathrm{t}_{\mathrm{r}}$ os intervalos de tempo de acionamento das válvulas $\mathrm{V}_{1} \mathrm{e} \mathrm{V}_{3}$, respectivamente. 
A inserção seqüencial de uma alíquota da solução da amostra seguida de uma alíquota da solução do reagente é chamada de ciclo de amostragem ${ }^{13}$.

As vazões das soluções transportadora (Ca), da amostra (A) e do reagente $(\mathrm{R})$ foram previamente estabelecidas em 2,6; 2,6 e 1,5 mL $\min ^{-1}$, respectivamente. Também os intervalos de tempo $\left(\mathrm{t}_{\mathrm{a}}, \mathrm{t}_{\mathrm{r}}\right)$ de acionamento das válvulas $\mathrm{V}_{1}$ e $\mathrm{V}_{3}$ e a temperatura do reator foram fixados em $0,5 \mathrm{~s}$ e $70{ }^{\circ} \mathrm{C}$, respectivamente. Esta temperatura foi obtida aplicando-se uma diferença de potencial de 7,0 V no reator de $50 \mathrm{~cm}$ $(460 \mu \mathrm{L})$. Assim, as variáveis estudadas foram o número de ciclos de amostragem e a concentração da solução de periodato de sódio.

$\mathrm{O}$ número de ciclos de amostragem pode ser repetido quantas vezes for necessário a fim de se obter a melhor sensibilidade. Para otimizar o volume da amostra, foram realizados experimentos variando o número de ciclos de 4 a 14, mantendo-se constantes os demais parâmetros. Durante o transporte da zona de amostra através do reator ocorria a mistura concomitante entre as alíquotas das soluções da amostra e do reagente e o aquecimento, favorecendo o desenvolvimento da reação. O produto da reação foi detectado a $548 \mathrm{~nm}$ e o perfil do sinal gerado em função do tempo era traçado no vídeo do computador, permitindo a visualização em tempo real.

Para definir a concentração do reagente, foram efetuados experimentos usando soluções de periodato de sódio de 6,0; 8,0 e 10,0\% $(\mathrm{m} / \mathrm{v})$. Nestes testes foram programados 12 ciclos de amostragem. Experimentos também foram realizados para otimizar o volume de reagente, variando o intervalo de tempo de acionamento das válvulas de 0,25 a 1,5 s, permitindo uma variação de volume de $6,25 \mu \mathrm{L}$ a $37,5 \mu \mathrm{L}$

Em virtude do sistema de aquecimento proposto, ocorria a liberação de bolhas de gás e sua intensidade aumentava com a temperatura da solução. A presença de bolhas de gás na cela de fluxo causa erro de leitura, então, para evitar este efeito foi acoplada uma câmara para remoção das bolhas, conforme indicado no diagrama de fluxos da Figura 2.

O tempo de residência da zona da amostra era em torno de $11 \mathrm{~s}$, então na execução dos experimentos descritos foi programada a interrupção do bombeamento durante $60 \mathrm{~s}$ para permitir o desenvolvimento da reação. Para isso, no final da etapa de amostragem, o computador enviava através da interface serial um comando para a bomba peristáltica interromper o bombeamento. Visando otimizar também este tempo, foram realizados os experimentos descritos a seguir. Ao terminar a etapa de amostragem eram interrompidos ambos, o fornecimento de energia para o aquecedor e a rotação da bomba peristáltica. Os testes foram efetuados variando o intervalo de tempo de parada de bombeamento de 30 a $150 \mathrm{~s}$ com incrementos de $30 \mathrm{~s}$. Para isso, foram usadas soluções de $\mathrm{Mn}^{2+} 20 \mathrm{mg} \mathrm{L}^{-1}$ e $\mathrm{NaIO}_{4} 8,0 \%$ $(\mathrm{m} / \mathrm{v})$ com 12 ciclos de amostragem e a temperatura mantida em $70{ }^{\circ} \mathrm{C}$.

Depois de finalizados os estudos das variáveis do sistema, determinou-se $\mathrm{Mn}^{2+}$ em um conjunto de amostras de digeridos de plantas, visando demonstrar a aplicabilidade do sistema.

\section{RESULTADOS E DISCUSSÃO}

\section{Estudos relacionados à resposta do aquecedor}

O princípio de funcionamento do micro-aquecedor era semelhante ao de um calorímetro de fluxo contínuo, assim, a temperatura da solução na saída do reator era função da vazão e da diferença de potencial aplicada. Observa-se na Figura 1, que o fio de aquecimento está ligado à saída de 6 volts. $\mathrm{O}$ "dimmer" (Dm) colocado no circuito de entrada permite selecionar diferenças de potencias mais baixas. Este arranjo permitiu o ajuste da temperatura requerida para o desenvolvimento da reação sem alterar as vazões das soluções utilizadas no procedimento analítico.

Dependendo das condições de isolamento térmico pode haver perda para o ambiente e a temperatura na saída pode ser mais baixa do que a esperada. Então, visando verificar a resposta do aquecedor em função da vazão do fluido trocador de calor e da diferença de potencial aplicada, foram feitos experimentos com três reatores de diferentes dimensões e os resultados são mostrados na Figura 3 e na Tabela 1.

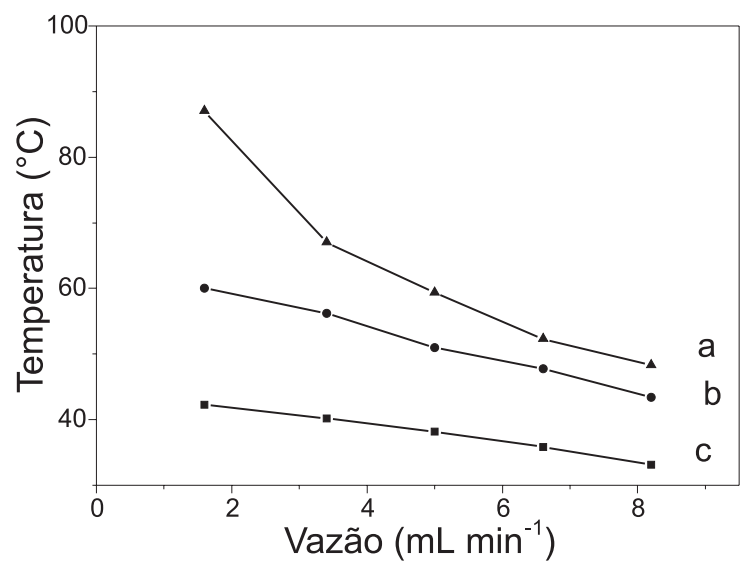

Figura 3. Efeito da vazão sobre a temperatura. As curvas $a, b$ e $c$ correspondem às diferenças de potenciais de 7,0; 5,0 e 3,0 V, respectivamente; $B=$ reator, volume interno de $460 \mu \mathrm{L}$; resistência elétrica de $3,4 \Omega ;$ vazão $=8,7 \mathrm{~mL} \mathrm{~min}^{-1}$ e temperatura inicial do fluido transportador $T_{o}=22,8^{\circ} \mathrm{C}$

Tabela 1. Efeito da vazão e da diferença de potencial

\begin{tabular}{cccc}
\hline $\begin{array}{c}\text { Vazão } \\
\left(\mathrm{mL} \mathrm{min}^{-1}\right)\end{array}$ & $\begin{array}{c}\text { Voltagem } \\
(\mathrm{V})\end{array}$ & $\begin{array}{c}\text { Reator } 1 \\
\text { Temperatura }\left({ }^{\circ} \mathrm{C}\right)\end{array}$ & $\begin{array}{c}\text { Reator } 2 \\
\text { Temperatura }\left({ }^{\circ} \mathrm{C}\right)\end{array}$ \\
\hline 0,9 & 2,0 & 86,0 & 51,7 \\
1,6 & 2,0 & 72,7 & 49,6 \\
3,4 & 2,0 & 57,3 & 39,0 \\
5,0 & 2,0 & 49,8 & 34,2 \\
6,6 & 2,0 & 44,5 & 32,6 \\
8,2 & 2,0 & 40,0 & 32,4 \\
0,9 & 3,0 & - & 87,6 \\
1,6 & 3,0 & 91,9 & 74,5 \\
3,4 & 3,0 & 82,3 & 57,4 \\
5,0 & 3,0 & 70,3 & 54,0 \\
6,6 & 3,0 & 61,1 & 49,4 \\
8,2 & 3,0 & 54,1 & 45,6 \\
0,9 & 3,5 & - & 89,8 \\
1,6 & 3,5 & - & 82,1 \\
3,4 & 3,5 & 91,1 & 68,0 \\
5,0 & 3,5 & 77,4 & 62,2 \\
6,6 & 3,5 & 68,7 & 60,9 \\
8,2 & 3,5 & 60,2 & 59,2 \\
\hline
\end{tabular}

Reator 1: volume de $90 \mu \mathrm{L}$ e resistência de $0,9 \Omega$; Reator 2: volume de $200 \mu \mathrm{L}$ e resistência de $1,7 \Omega$; Temperatura inicial da água: $22{ }^{\circ} \mathrm{C}$

De acordo com os resultados obtidos com reator de $55 \mathrm{~cm} \mathrm{de}$ comprimento e as diferenças de potenciais de 3,0 e 5,0 V, a temperatura decresce com o aumento da vazão do fluido de forma praticamente linear. Este tipo de resposta era esperado, entretanto, para a diferença de potencial de 7,0 V esta tendência não foi observada entre as vazões de 1,6 e 3,4 $\mathrm{mL} \mathrm{min}{ }^{-1}$. Empregando a vazão de 
1,6 mL $\min ^{-1}$ a temperatura observada foi de $87^{\circ} \mathrm{C}$. Nesta condição ocorre liberação de grande quantidade de bolhas de gás, segmentando a solução dentro do reator. A existência de um sistema bifásico (gás/líquido) dentro do reator alterava a resposta do mesmo.

Nos experimentos realizados com os reatores de 12 e de $21 \mathrm{~cm}$ de comprimento (Tabela 1) observa-se tendência semelhante para as três diferenças de potenciais. Embora os três reatores tivessem geometrias semelhantes, tinham dimensões distintas. Portanto, temperaturas mais baixas eram esperadas com os reatores mais longos, pois o aumento da resistência do aquecedor diminuía a potência liberada.

A estabilidade do sistema com relação à temperatura foi avaliada mediante várias leituras de uma solução de $\mathrm{Mn}^{2+} 20,0 \mathrm{mg} \mathrm{L}^{-1}$ para diferentes valores de temperatura. Como mostrado na Tabela 2, obteve-se uma boa repetibilidade dos resultados. Na situação menos favorável, o desvio padrão relativo estimado foi de 1,2\%, o que indica boa estabilidade do sistema de aquecimento.

Tabela 2. Estabilidade do sistema com relação à temperatura

\begin{tabular}{cc}
\hline Temperatura $\left({ }^{\circ} \mathrm{C}\right)$ & RSD $(\%)$ \\
\hline $35,1 \pm 0,2$ & 0,6 \\
$50,2 \pm 0,6$ & 1,2 \\
$60,8 \pm 0,5$ & 0,9 \\
$70,3 \pm 0,8$ & 1,2 \\
$80,4 \pm 0,4$ & 0,5 \\
\hline
\end{tabular}

Resultados: média de três leituras tomadas em intervalos de $15 \mathrm{~s}$.

\section{Otimização das variáveis do sistema}

O efeito do volume da zona de amostra no sinal analítico foi avaliado variando o número de ciclos de amostragem entre 4 e 14, correspondente aos volumes de 138 a $483 \mu \mathrm{L}$ com soluções de $\mathrm{Mn}^{2+}$ $20 \mathrm{mg} \mathrm{L}^{-1} \mathrm{e}$ do branco. A partir do volume de $400 \mu \mathrm{L}$ (12 ciclos de amostragem) não se observou aumento significativo na leitura do sinal, indicando que a zona da amostra praticamente alcançou a condição de volume infinito. Este efeito poderia ser esperado tendo em vista que o volume do reator era de $460 \mu \mathrm{L}$, assim, o volume de $400 \mu \mathrm{L}$ foi selecionado para os estudos posteriores.

Na Figura 4 é mostrado o efeito da concentração de $\mathrm{NaIO}_{4}$ na magnitude do sinal, onde se observa que a melhor resposta foi obtida com a concentração de $8,0 \%(\mathrm{~m} / \mathrm{v})$. A curva $c$ indica que, usando a solução de $\mathrm{NaIO}_{4}$ com a concentração de $10,0 \%$ (m/v), os resultados foram menos favoráveis. Não encontramos uma explicação apropriada para esse comportamento, entretanto experimentos repetidos nas mesmas condições sempre deram resultados semelhantes. Em vista destes resultados selecionou-se a concentração de $\mathrm{NaIO}_{4}$ de $8,0 \%(\mathrm{~m} / \mathrm{v})$. No item anterior foram estabelecidos 12 ciclos de amostragem como referência para os experimentos posteriores, assim, neste experimento, o volume do reagente foi variado aumentando o intervalo de tempo de acionamento da válvula $\mathrm{V}_{3}$ (Figura 2). A partir da curva $a$ selecionou-se o volume de $150 \mu \mathrm{L}$ como ótimo, que correspondia ao acionamento da válvula $\mathrm{V}_{3}$ durante $0,5 \mathrm{~s}$ em cada ciclo de amostragem, inserindo uma alíquota de $12,5 \mu \mathrm{L}$ da solução do reagente após cada inserção da alíquota da solução da amostra.

Os resultados discutidos nos itens precedentes foram obtidos interrompendo o bombeamento da solução transportadora durante $60 \mathrm{~s}$ para permitir o desenvolvimento da reação. Visando otimizar este parâmetro, foram efetuados testes variando o intervalo de tempo de 30 a $150 \mathrm{~s}$. Os resultados mostrados na Figura 5 indicam que a partir de $30 \mathrm{~s}$ o sinal do produto da reação permanecia praticamente constante. Entretanto, observa-se aumento do sinal referente ao branco, em vista disso, o intervalo de tempo de $30 \mathrm{~s}$ foi estabelecido.
Tendo estabelecido as condições operacionais, $\mathrm{Mn}^{2+}$ foi determinado em um conjunto de amostras e os resultados são mostrados na Tabela 3. A exatidão foi averiguada comparando-se os resultados com os obtidos por espectrometria de emissão óptica com plasma acoplado indutivamente. Aplicando-se o teste-t pareado encontrou-

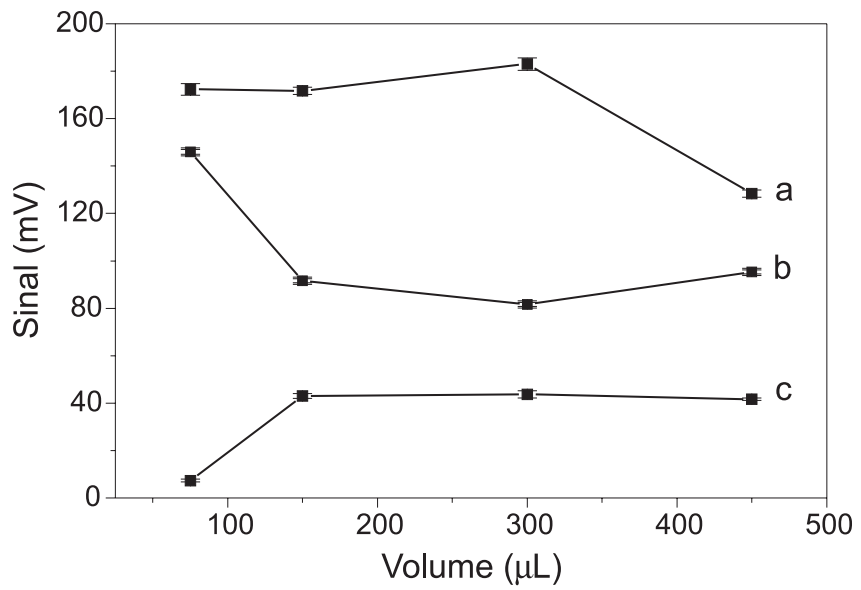

Figura 4. Efeito da concentração do reagente. As curvas a, b e c correspondem às concentrações de $\mathrm{NaIO}_{4} 8,0 \% ; 6,0 \%$ e 10,0\% ( $\left.\mathrm{m} / \mathrm{v}\right)$, respectivamente; diferença de potencial aplicada, 7,0 V; vazão $=6,7 \mathrm{~mL} \mathrm{~min}^{-1}$; volumes das soluções da amostra e do reagente 264 e $150 \mu \mathrm{L}$, respectivamente; Temperatura do reator $70{ }^{\circ} \mathrm{C}$. Os demais parâmetros foram os mesmos da Figura 3

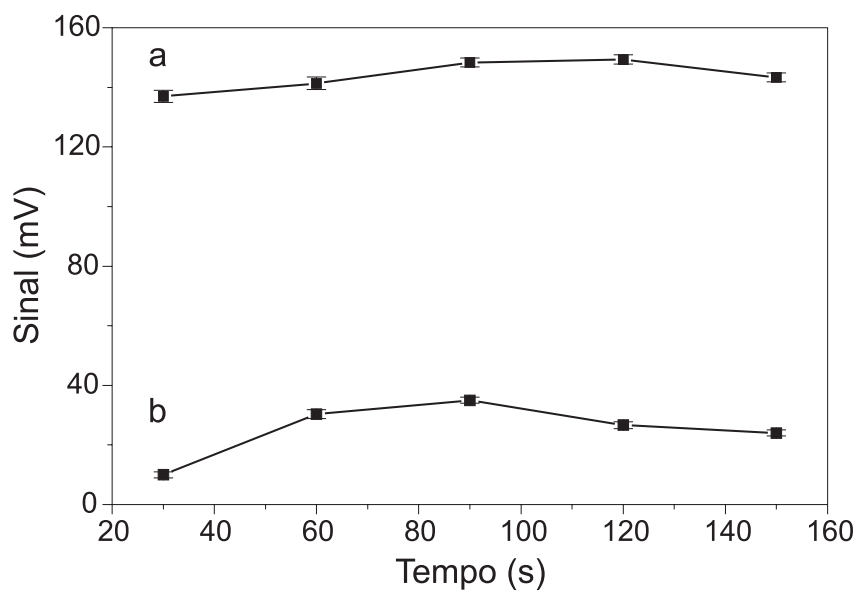

Figura 5. Efeito do tempo de residência sobre a reação. A curva a corresponde a uma solução de $\mathrm{Mn}^{2+}$ contendo $20,0 \mathrm{mg} \mathrm{L}^{-1}$; a curva b corresponde à leitura da solução do branco. Os outros parâmetros experimentais foram mantidos os mesmos da figura anterior

Tabela 3. Comparação dos resultados

\begin{tabular}{lccr}
\hline Amostra & $\begin{array}{c}\text { ICP-OES } \\
(\mu \mathrm{g} / \mathrm{g})\end{array}$ & $\begin{array}{c}\text { Sistema proposto } \\
(\mu \mathrm{g} / \mathrm{g})\end{array}$ & $(\%)$ \\
\hline Soja 1 & $1080 \pm 4$ & $909 \pm 5$ & 118 \\
Soja 2 & $1883 \pm 9$ & $2066 \pm 85$ & 91 \\
Soja 3 & $1152 \pm 14$ & $1081 \pm 21$ & 106 \\
Soja 4 & $1934 \pm 9$ & $2160 \pm 68$ & 89 \\
Soja 5 & $1917 \pm 11$ & $1515 \pm 55$ & 126 \\
Soja 6 & $1871 \pm 22$ & $1670 \pm 39$ & 112 \\
Café & $356 \pm 3$ & $340 \pm 31$ & 104
\end{tabular}

Resultados: média de três determinações consecutivas. Valores calculados para a matéria seca. test- $t$ pareado: valor calculado $=$ 0,$78 ;$ valor tabelado $=1,94$ 
se o valor de 0,78 . O valor tabelado é 1,94 , portanto não há diferença significativa em nível de confiança de $90 \%$. Outras características favoráveis, tais como freqüência analítica de 22 determinações por hora, consumo de reagente de $8,3 \mathrm{mg} \mathrm{NaIO}_{4}$ por determinação, e limite de detecção de $1,2 \mathrm{mg} \mathrm{L}^{-1}$ foram também obtidas.

\section{CONCLUSÕES}

Os resultados obtidos com esse sistema demonstram boa precisão e exatidão do procedimento, bem como baixo consumo de reagentes. $\mathrm{O}$ dispositivo para aquecimento pode ser facilmente construído. Além disso, a miniaturização do sistema, o acoplamento direto do reator de aquecimento ao módulo de análise, e o baixo consumo de energia são algumas vantagens apresentadas em relação aos equipamentos comerciais de grande porte.

A temperatura de $70{ }^{\circ} \mathrm{C}$ foi facilmente obtida aplicando uma diferença de potencial de 7,0 V, permitindo que em $30 \mathrm{~s}$ a reação se completasse, o que é vantajoso considerando-se que à temperatura de $25^{\circ} \mathrm{C}$, a reação demora 15 min para alcançar o equilíbrio ${ }^{10}$.

\section{AGRADECIMENTOS}

Os autores agradecem à FAPESP, CAPES, CNPq e PRONEX pelo apoio financeiro recebido.

\section{REFERÊNCIAS}

1. Ruzicka, J.; Hansen, E. H.; Anal. Chim. Acta 1975, 78, 145.

2. Ruzicka, J.; Hansen, E. H.; Anal. Chim. Acta 1979, 106, 207.

3. Krug, F. J.; Reis, B. F.; Giné, M. F.; Ferreira, J. R.; Jacintho, A. O.; Zagatto, E. A. G.; Anal. Chim. Acta 1983, 151, 39.

4. Pasquini, C.; Oliveira, W. A.; Anal. Chem. 1985, 57, 2575.

5. Pereira, A. V.; Aniceto, C.; Fatibello-Filho, O.; Analyst 1998, 123, 1011.

6. Oliveira, A. F.; Fatibello-Filho, O.; Talanta 1999, 50, 899.

7. Oliveira, A. F.; Fatibello-Filho, O.; Nóbrega, J. A.; Talanta 2001, 55, 677.

8. Mesquita, M.; Jacintho, A. O.; Zagatto, E. A. G.; J. Braz. Chem. Soc. 1990, 1,28 .

9. Cruz Vieira, I.; Fatibello-Filho, O.; Analyst 1998, 123, 1809.

10. Smiderle, M.; Reis, B. F.; Rocha, F. R. P.; Anal. Chim. Acta 1999, 386, 129.

11. Marczenko, Z.; Separation and Spectrophotometric Determination of Elements, Ellis Horwood: Chichester, 1986.

12. Onishi, H.; Photometric Determination of Traces of Metals, 4th ed., John Wiley \& Sons: New York, 1989.

13. Reis, B. F.; Giné, M. F.; Zagatto, E. A. G.; Lima, J. L. F. C.; Lapa, R. A.; Anal. Chim. Acta 1994, 293, 129.

14. Fernandes, R. N.; Reis, B. F.; Talanta 2002, 58, 1.

15. Krug, F. J.; Bergamin $\mathrm{F}^{\mathrm{O}}$, H.; Zagatto, E. A. G.; Jorgensen, S.S.; Analyst 1977, 102, 503 . 